Supporting information for:

\title{
Molecular networking of high-resolution tandem ion mobility spectra: a structurally relevant way of organizing data in glycomics?
}

Part 1: Supplementary Texts and Figures

Simon Ollivier, Mathieu Fanuel, Hélène Rogniaux, ${ }^{*}$ and David Ropartz

INRAE, UR BIA, F-44316 Nantes, France

INRAE, BIBS Facility, F-44316 Nantes, France

Correspondence to: helene.rogniaux@inrae.fr

$$
\text { +33(0) } 240675034
$$

\section{Table of Content}

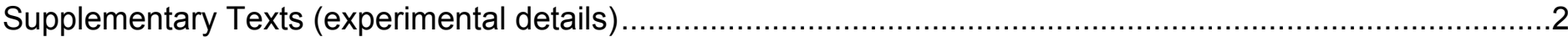

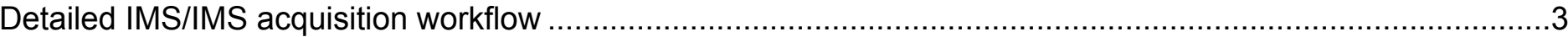

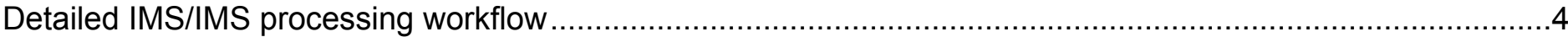

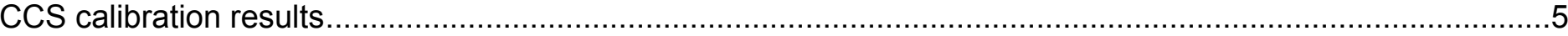

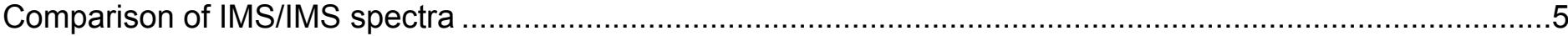

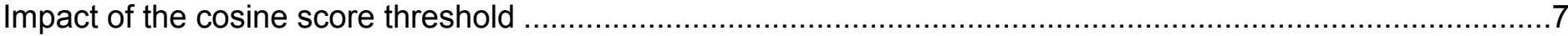

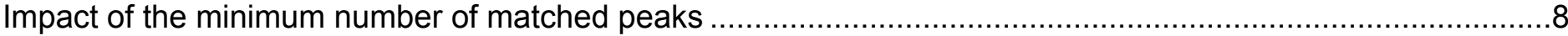

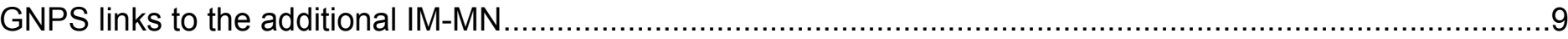

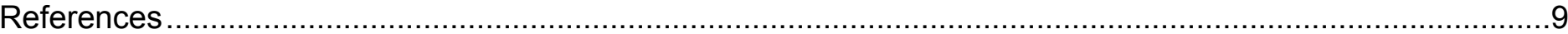

Supplementary Tables Attached $x$ Isx file 
Supplementary text 1 . Mass spectrometry parameters.

Oligosaccharides were analyzed using positive ion mode electrospray, as singly- or doubly-charged lithium adducts. The electrospray parameters were: capillary $3.0 \mathrm{kV}$; cone $60 \mathrm{~V}$; source offset $10 \mathrm{~V}$; source temperature $100{ }^{\circ} \mathrm{C}$; desolvation temperature $300^{\circ} \mathrm{C}$; cone gas $0 \mathrm{~L} / \mathrm{h}$; desolvation gas $600 \mathrm{~L} / \mathrm{h}$; nebulizer gas 6.0 Bar $(P=$ 9 e-3 mBar). Any change to these parameters (e.g. to increase MS signal intensity) is given in the 'comments' in Table S3.

Mass-selection was performed in the quadrupole before ion mobility separation, on the $\mathrm{m} / \mathrm{z}$ ratios given in Table S3 (LM resolution: 4.9; HM resolution: 20; ion energy $0.4 \mathrm{~V}$; Pre-filter $2.0 \mathrm{~V}$ ).

The voltages and pressures were:

- $\quad$ Before the IMS cell:

- Stepwave (ion optics): body gradient 20V; head gradient 10V; ion guide (IG) 1 offset $3.0 \mathrm{~V}$; IG 2 offset $0.3 \mathrm{~V}$; IG TW velocity $150 \mathrm{~m} / \mathrm{s}$; IG TW pulse height $0.4 \mathrm{~V}$.

- Trap: trap TW velocity $300 \mathrm{~m} / \mathrm{s}$; trap TW pulse height $1.0 \mathrm{~V}$; trap entrance 2.0; trap bias $2.0 \mathrm{~V}$; trap DC $-4.0 \mathrm{~V}$; trap exit $0.0 \mathrm{~V}$; post trap gradient $7.0 \mathrm{~V}$; post trap bias $35.0 \mathrm{~V}$; collision gas $=\mathrm{N}_{2}$ $3.5 \mathrm{~mL} / \mathrm{min}(\mathrm{P}=2.5 \mathrm{e}-2 \mathrm{mBar})$.

- $\quad$ Helium cell: entrance $5.0 \mathrm{~V}$; bias $20.0 \mathrm{~V}$; exit DC -10.0 V (P = $2.1 \mathrm{mBar})$.

- $\quad$ After the IMS cell:

- $\quad$ Transfer: pre transfer guide gradient $7.0 \mathrm{~V}$; pre trans bias $0.0 \mathrm{~V}$; transfer entrance $2.0 \mathrm{~V}$; transfer gradient $0.8 \mathrm{~V}$; transfer exit $15 \mathrm{~V}$.

- $\quad$ TOF analyzer: $\mathrm{V}$-mode (sensitivity mode), $\mathrm{P}=1.5 \mathrm{e}-7 \mathrm{mBar}$.

Supplementary text 2 . Ion mobility spectrometry parameters.

Cyclic IMS experiments were performed in N60 purity nitrogen (Alphagaz 2, Air Liquide, France), at a pressure of $1.7 \mathrm{mbar}$ (measured as described in Giles et $\mathrm{al}^{1}$ ). The traveling wave parameters were as follows: wave height $16 \mathrm{~V}$, wave velocity $350 \mathrm{~m} / \mathrm{s}$.

The cyclic ion mobility geometry was used to perform tandem IMS (IMS/IMS) experiments. A reverse traveling wave ejects the ions in a defined arrival time window backwards to a pre-array store cell (= pre-store). CID fragmentation can the be performed upon reinjection of the ions in the IMS cell by changing specific voltages, forming a gradient used to increase the kinetic energy of the ions:

- $\quad$ The pre-array gradient is increased (default $=85 \mathrm{~V}$ )

- $\quad$ The pre-array bias is increased, kept $15 \mathrm{~V}$ below the pre-array gradient (default $=70 \mathrm{~V}$ )

- $\quad$ The array offset is decreased (default $=45 \mathrm{~V}$ )

IMS/IMS fragmentation energies are noted "gradient $\rightarrow$ bias $\rightarrow$ offset" throughout the paper (e.g. $85 \rightarrow 70 \rightarrow 45$ ). 
Supplementary Figure 1. Detailed scheme of an IMS/IMS experiment. A. The array region allows to select and fragment a precursor ion, using a sequence of events (see Table S3). B. The multifunction array region is used to set the direction of the ion path. The traveling wave can be directed forward for ejection and TOF detection (yellow), sideways for separation (orange), or backwards for selection to the pre-store cell (red). C. Potential energy diagrams showing the use of the pre-store cell for selection and fragmentation of ions. (Figure adapted from Ollivier et al. ${ }^{2}$ )

A.

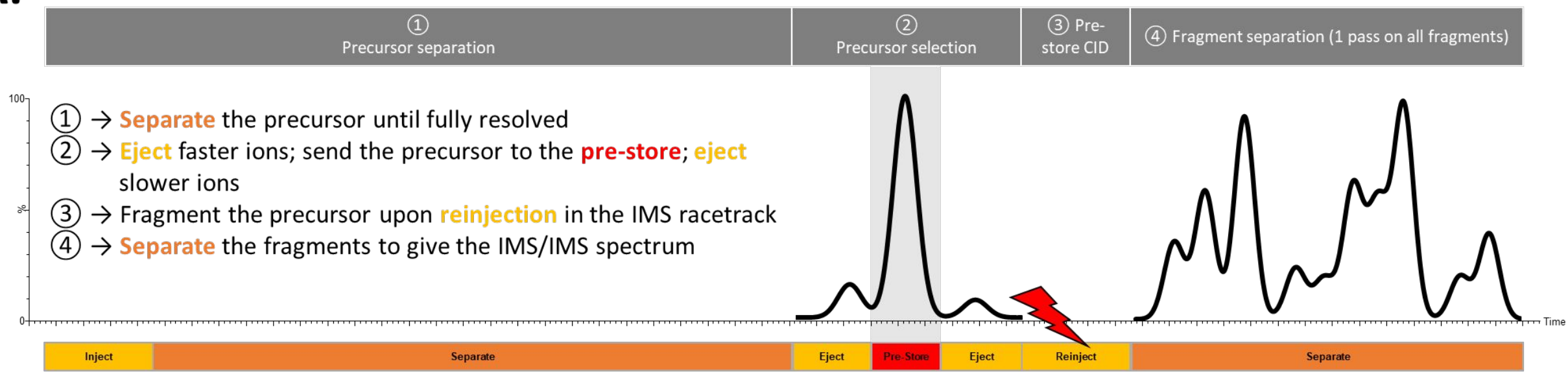

B.

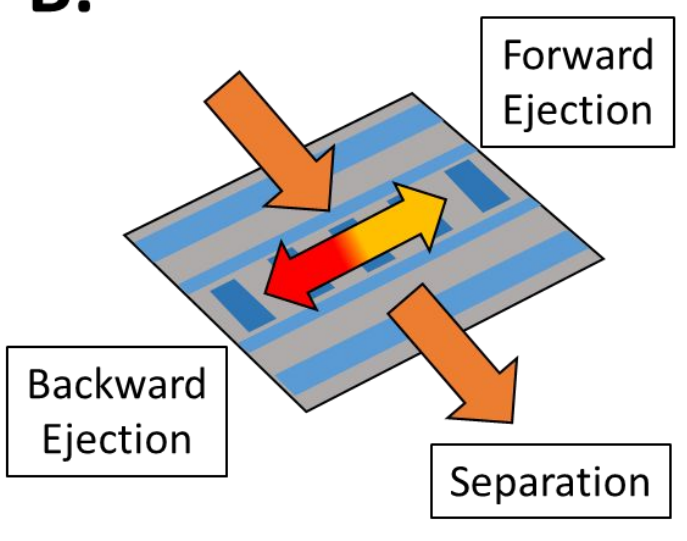

C.

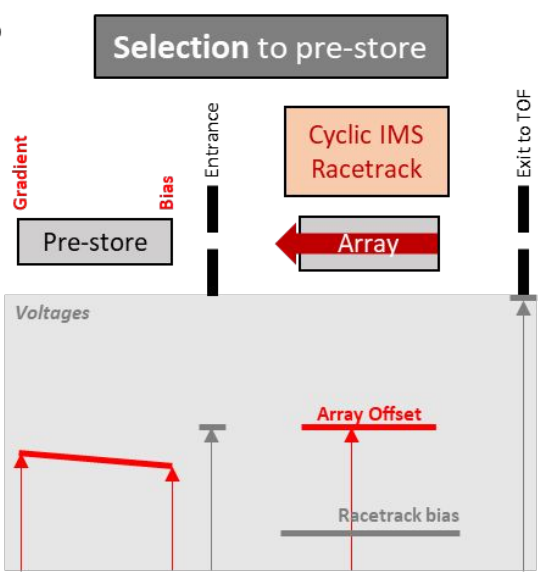

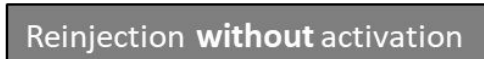
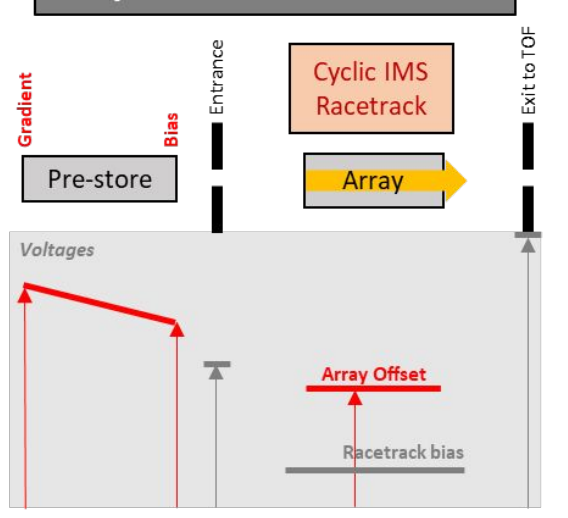

Reinjection with activation

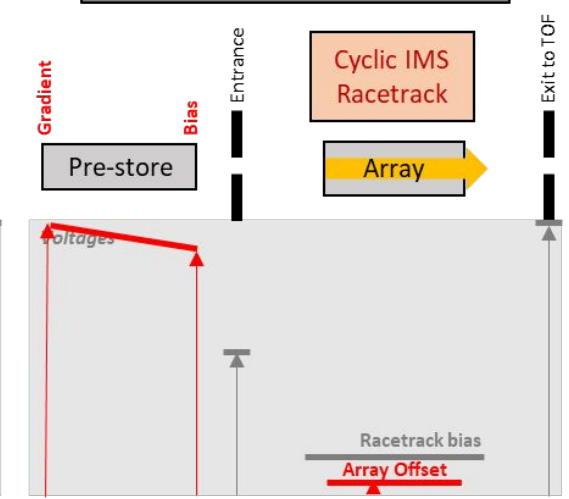


Supplementary Figure 2. Data processing workflow, from the generation of the data file in the manufacturer format to the creation of the MN.

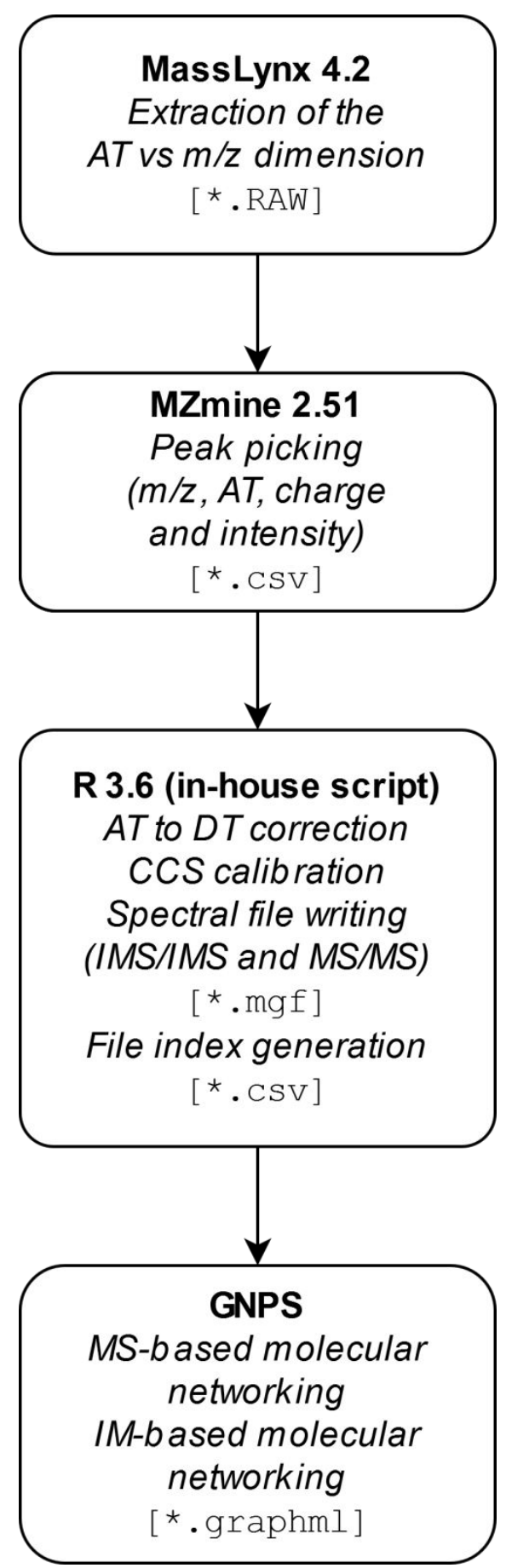


Supplementary Figure 3. Results of the $\log { }^{\top}{ }^{W} C C S_{\mathrm{N}^{2}}$ calibration, with the $R^{2}$ for $\ln \left(\mathrm{dt}^{\prime}\right) \mathrm{vs} \ln (\mathrm{CCS})$ and the mean CCS deviation between literature values and recalculated values. The interactive html plot with $\mathrm{m} / \mathrm{z}$ and ${ }^{{ }^{T W}} \mathrm{CCS}_{\mathrm{N} 2}$ values for each calibration point is available at: https://osf.io/ne7wd/.

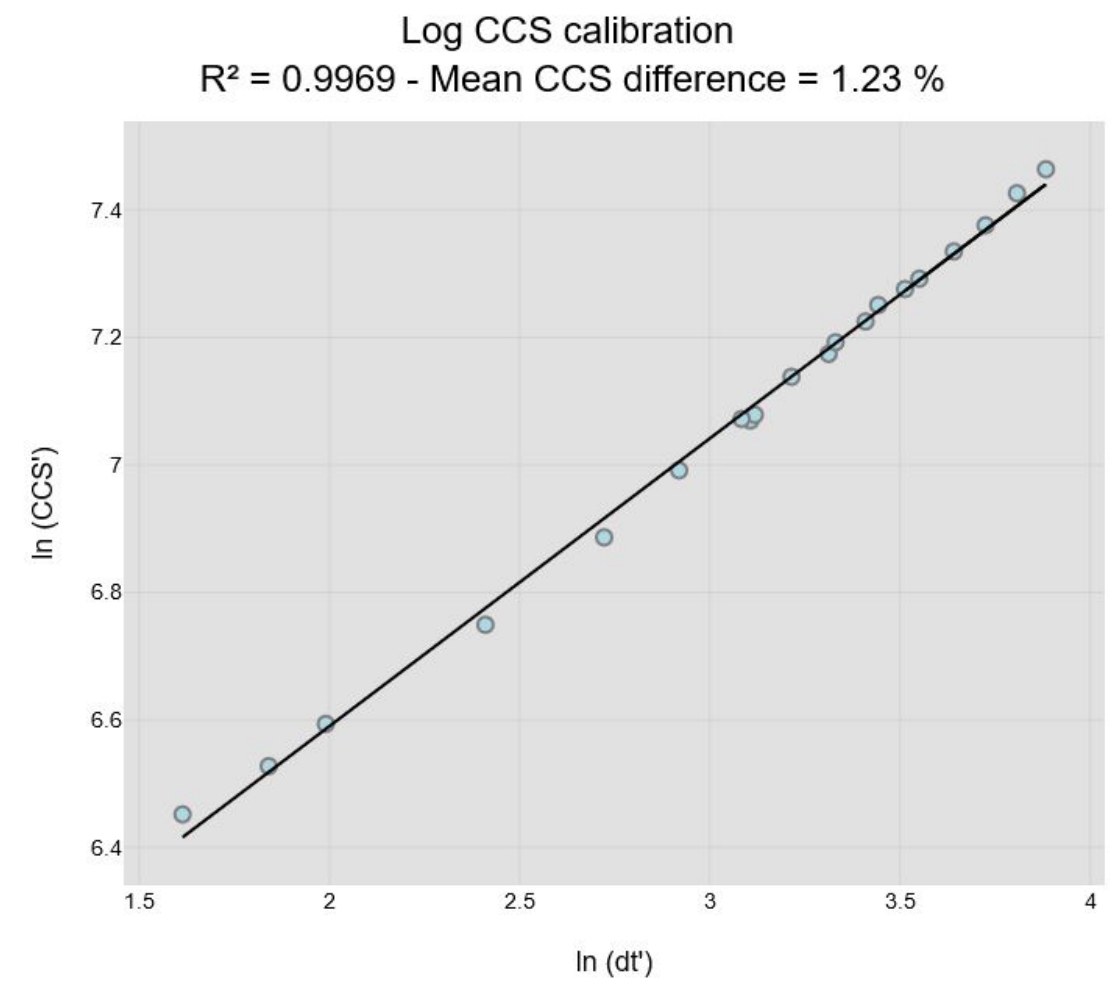

Supplementary Figure 4. Comparison of two centroided IMS/IMS spectra for clusters la (maltopentaose, bottom) and lb (Dextran DP5, top).

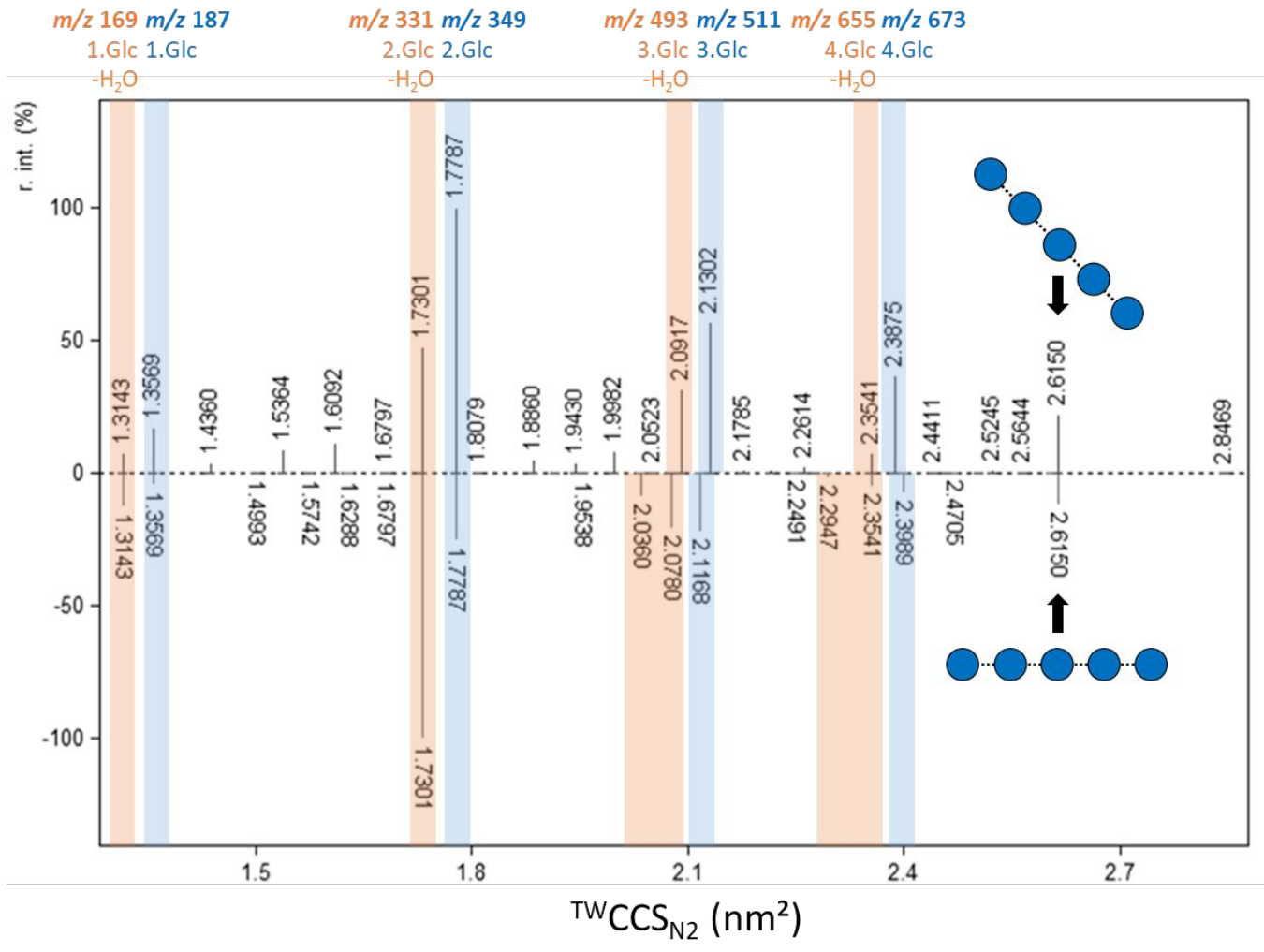


Supplementary Figure 5. Arrival time distributions of the fragments of A. cellopentaose and B. maltopentaose as displayed by MZmine before they were mass-deconvolved and calibrated to CCS values to give IMS/IMS spectra.
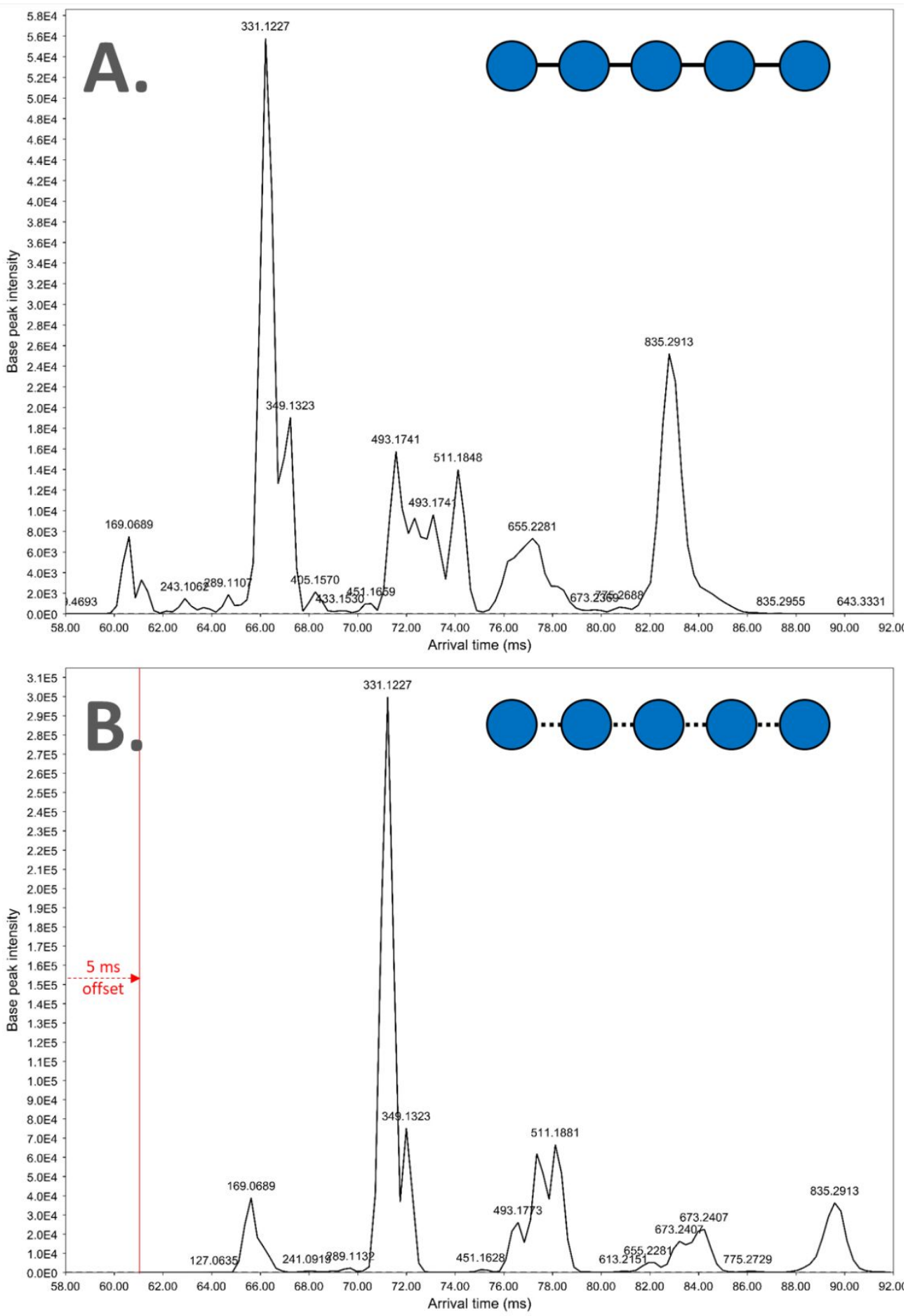
Supplementary Figure 6. Impact of the cosine score threshold on the topology of the network. A-E Reanalyses of the extended IM-MN from the main text in which two ions are clusterized if they have a minimum of 8 shared peaks and a cosine score above A. 0.5 , B. 0.6, C. 0.7, D. 0.8, and E. 0.9; F. variation of the number of edges between nodes as a function of the cosine score threshold.
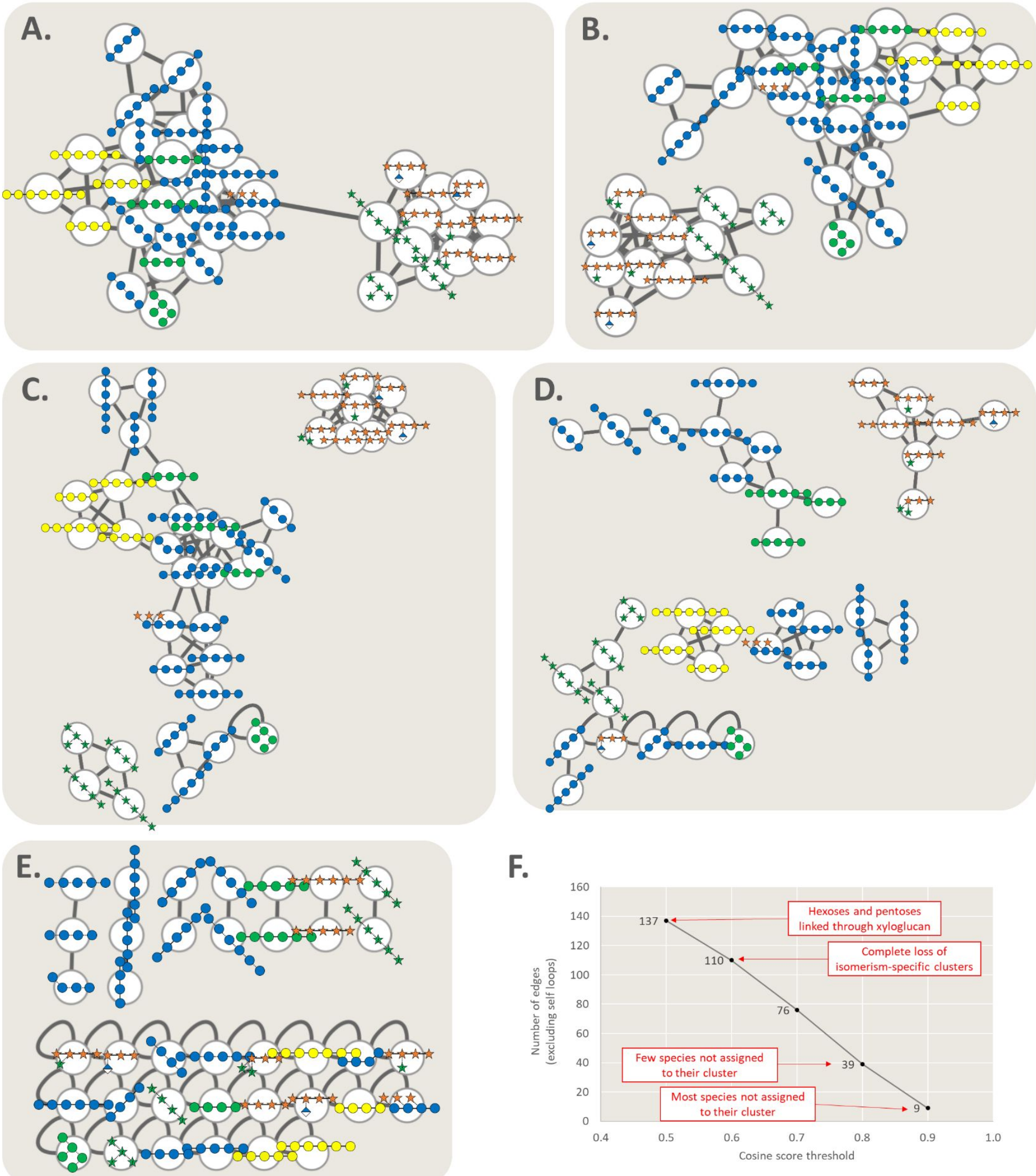
Supplementary Figure 7. Impact of the minimum number of shared peaks on the topology of the network. A-E Reanalyses of the extended IM-MN from the main text in which two ions are clusterized if they have a cosine score above 0.75 and a minimum number of shared peaks of: A. 5, B. 6, C. 7, D. 9, and E. 10; F. variation of the number of edges between nodes as a function of the minimum number of shared peaks.
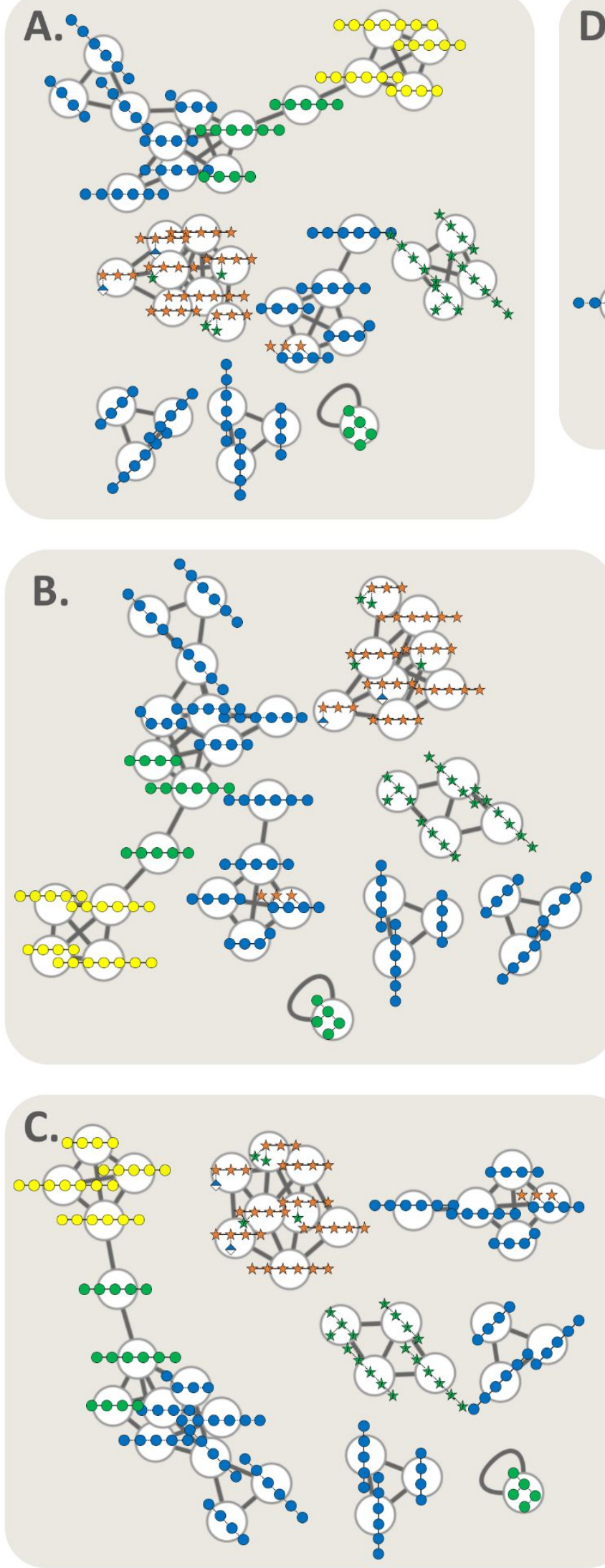
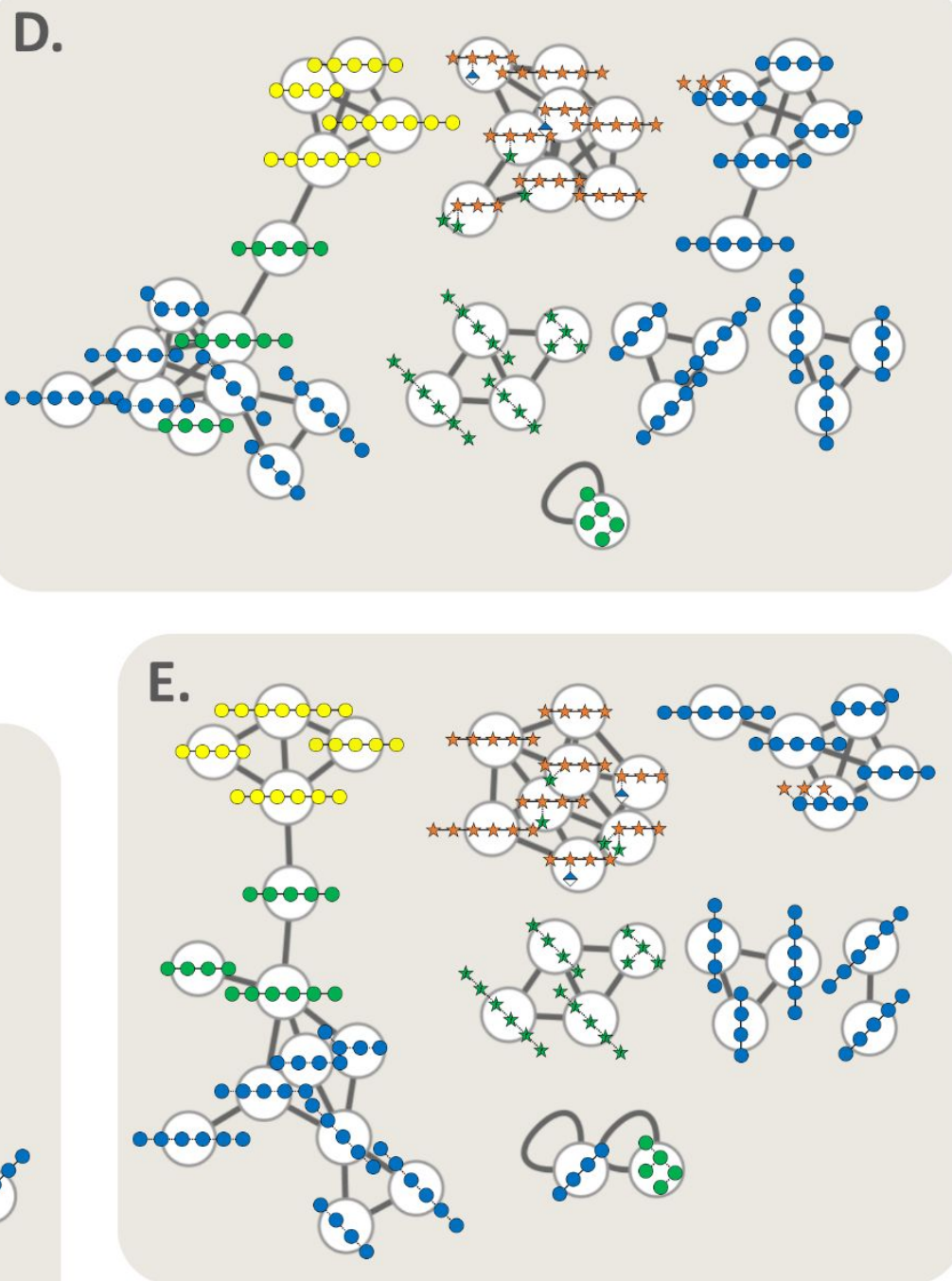

F.

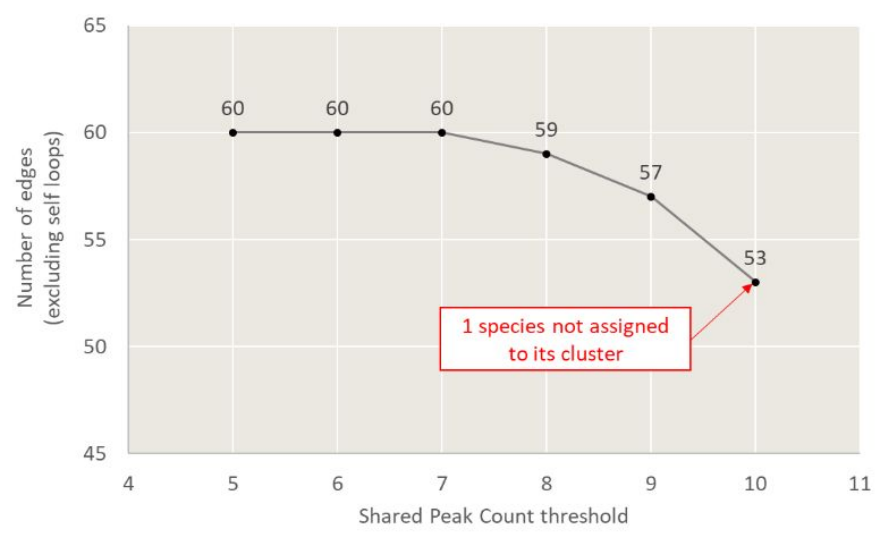




\section{Supporting data availability}

The molecular networks used to study the impact of the cosine score threshold (Suppl. Fig. 4) can be downloaded at:

\begin{tabular}{|c|l|}
\hline $\begin{array}{c}\text { Cosine } \\
\text { threshold }\end{array}$ & GNPS link \\
\hline 0.5 & https://gnps.ucsd.edu/ProteoSAFe/status.jsp?task=7ccbcf39e0f64eaabe1d0bbac77bf4e4 \\
\hline 0.6 & https://gnps.ucsd.edu/ProteoSAFe/status.jsp?task=88c1afdbee144331b5a8ee13cf24e7ac \\
\hline 0.7 & https://gnps.ucsd.edu/ProteoSAFe/status.jsp?task=984ab0f8e89848478ad284d14237fcba \\
\hline 0.8 & https://gnps.ucsd.edu/ProteoSAFe/status.jsp?task=202667be8b5a48e4ad2eddb24d9ded16 \\
\hline 0.9 & $\underline{\text { https://gnps.ucsd.edu/ProteoSAFe/status.jsp?task=e0c7a4db604e4c04a9bbad1e52e6da8f }}$ \\
\hline
\end{tabular}

The molecular networks used to study the impact of the min. number of shared peaks (Suppl. Fig. 5) can be downloaded at:

\begin{tabular}{|c|l|}
\hline $\begin{array}{c}\text { Min. shared } \\
\text { peaks }\end{array}$ & GNPS link \\
\hline 5 & https://gnps.ucsd.edu/ProteoSAFe/status.jsp?task=db5f715ebf2c43928c232ec7013c47a7 \\
\hline 6 & https://gnps.ucsd.edu/ProteoSAFe/status.jsp?task=f15a65111fcd43bf9ecd782cd2cc77d8 \\
\hline 6 & https://gnps.ucsd.edu/ProteoSAFe/status.jsp?task=ea919ae9997e4e0d8ea01ef182d5c197 \\
\hline 8 & See main text \\
\hline 9 & https://gnps.ucsd.edu/ProteoSAFe/status.jsp?task=3299976989504daea95934b30706dd60 \\
\hline 10 & https://gnps.ucsd.edu/ProteoSAFe/status.jsp?task=192969a0369b47f496ced3ce55c80077 \\
\hline
\end{tabular}

\section{References}

1. Giles, K. et al. A Cyclic Ion Mobility-Mass Spectrometry System. Anal. Chem. 91, 8564-8573 (2019).

2. Ollivier, S. et al. Anomeric Retention of Carbohydrates in Multistage Cyclic lon Mobility (IMSn): De Novo Structural Elucidation of Enzymatically Produced Mannosides. Anal. Chem. 93, 6254-6261 (2021). 